\title{
Discrepancy Between Normal Tension Glaucoma and Primary Open Angle Glaucoma with Confocal Microscopy Examination of Corneal Nerves
}

\section{Ziyuan Liu}

Peking University third hospital

Dalan Jing

peking university third hospital

Yilin Chou

Peking University Third Hospital

Haikun Wang

Peking University Third Hospital

\section{Shuang Gao}

Peking University Third Hospital

Wei Wang ( $\sim$ puh3_ww@bjmu.edu.cn )

Peking University Third Hospital https://orcid.org/0000-0003-0035-4813

\section{Research article}

Keywords: Corneal confocal microscopy, Normal tension glaucoma, Primary open angle glaucoma, Corneal subbasal nerve plexus, Neurodegenerative condition

Posted Date: May 8th, 2020

DOI: https://doi.org/10.21203/rs.3.rs-23241/v1

License: (a) This work is licensed under a Creative Commons Attribution 4.0 International License. Read Full License 


\section{Abstract \\ Background}

To investigate the morphological features of corneal subbasal nerve plexus (CSNP) and Langerhans cells (LCs) in normal tension glaucoma (NTG) and primary open angle glaucoma (POAG)

\section{Methods}

Ten eyes with NTG (8 untreated), 23 eyes with POAG ( 9 untreated) and 31 eyes of healthy subjects were recruited. CSNP and LCs were assessed by corneal confocal microscopy (CCM) and peripapillary retinal nerve fiber layer (RNFL) was measured with optical coherence tomography (OCT). CCM parameters including nerve fiber length $(\mathrm{FL})$, branch number $(\mathrm{BN})$, nerve width (NW), nerve reflectivity (NR), total and local nerve tortuosity (NT) as well as type 1 and type 2 LCs were compared across all groups, as well as between the topical medication treated and the nontreated patients.

\section{Results}

The NTG had the longest FL(3494.60 \pm 523.01$)$, most BN(19.78 \pm 5.56$)$, thinnest NW(2.96 \pm 1.20$)$, lowest reflectivity $(143.03 \pm 14.01)$ and most tortuosity $(1.09 \pm 0.07)$ compared with POAG and the normal. The POAG had similar CSNP to the normal. Same results were also found in untreated NTG and untreated POAG. Untreated NTG (3/8), untreated POAG(3/9) and treated POAG (7/14) had significantly more type 2 LCs than the normal (3/31). The treated POAG had longer FL (3240.71 \pm 627.81$)$, more BN(19.04 \pm 4.58$)$, NW(3.66 \pm 0.54$), N R(154.65 \pm$ 13.95), more NT $(1.07 \pm 0.03)$ than the nontreated but with no significant difference. BN had significant correlations with RNFL thickness in both NTG and POAG groups.

\section{Conclusions}

The NTG had characteristic CSNP quite different from that in the POAG and the normal, while the latter two shared more morphological features. The CCM parameters had associations with the RNFL thickness. Untreated glaucoma patients had obvious immature LCs indicating their relationship to ocular surface inflammation.

\section{Background}

Primary open angle glaucoma (POAG) is a multifactorial optic neuropathy, characterized by progressive thinning of retinal nerve fiber layer (RNFL) and peculiar visual field defect which could cause irreversible blindness ${ }^{1}$. Nearly 67 million people have the disease worldwide and the population is estimated to increase to 112 million by $2040^{1}$,

2. Elevated intraocular pressure (IOP) is a prominent risk factor for POAG progression and is the main target for treatment ${ }^{3}$. Accumulative exposure to high IOP impairs retinal ganglion cells (RGC) whose axons constitute the RNFL and optic projection to the brain ${ }^{4}$.

However, POAG's association with IOP is not exclusive. A large number of POAG patients have an IOP within normal range and are categorized as normal tension glaucoma $(N T G)^{5}$. NTG is a subtype of POAG manifesting 
RNFL defect and an open anterior chamber angle. NTG is more prevalent among Asian populations and its epidemiology varies ${ }^{6}$. Since its pressure-independent feature and unique disease course, a controversy exists that whether it should be regarded as a disease within POAG spectrum or as a distinctive disease ${ }^{7}$. The presence of typical glaucomatous optic nerve damage in NTG but with no clinical evidence of elevated IOP indicate other risk factors such as hemodynamic insufficiency ${ }^{8-10}$ and immunologic factors ${ }^{11}$.

Amid all of these efforts for exploring NTG pathomechanisms, diffuse brain damage in NTG has been noticed in recent decade ${ }^{12}$. A high coincidence between NTG and progressive sensorineural hearing loss as well as an association of NTG and dysgeusia have also been reported ${ }^{13}, 14$. Neurodegenerative changes beyond retina and optic nerve head suggest a possible pathogenesis of IOP-independent primary neural alterations in NTG.

However, most of the knowledge about NTG neuropathy beyond the eyeball has been derived from nerve functional analysis. Structural observation is limited due to the invisible features of these nerves in vivo. The transparent nature of cornea allows morphologic evaluation of the corneal nerves in vivo by the means of corneal confocal microscopy (CCM). The cornea is densely innervated by the ophthalmic division of the fifth cranial nerve, the trigeminal nerve. The corneal subbasal nerve fiber plexus (CSNP) which lies between basal epithelium and Bowman's membrane is the ending branch of ophthalmic nerve and shares the characteristics of small fiber nerves. The CSNP can be well studied by CCM and its degeneration has been discovered in many systemic neurodegenerative diseases such as Parkinson's disease, amyotrophic lateral sclerosis and diabetic peripheral neuropathy where CSNP alteration was associated with other nerve system degeneration ${ }^{15-19}$. The visibility of CSNP by CCM has gained growing popularity in researches as a disease severity predictor for the neurological disorders.

With CCM, we examined the CSNP and Langerhans cells (LCs) of NTG and POAG patients (with elevated IOP) and compared the structural changes of corneal nerves and then we explored the correlations between these anatomic alterations of corneal nerves and the glaucomatous changes of RNFL which is the most widely used parameter in clinical for measuring glaucoma progression. This study may add new knowledge to the current understanding of POAG and NTG neuropathy.

\section{Methods}

This prospective observational study consecutively recruited 33 eyes of 22 glaucoma patients (10 eyes with NTG, 23 eyes with POAG) seen in the Ophthalmology department of Peking University Third Hospital, Beijing, China from June 2017 to December 2017. Fourteen eyes of the POAG were under one to two kinds of antiglaucoma topical medication and the rest nine were newly diagnosed without treatment of any eye drops. In particular, eight eyes of the NTG were newly diagnosed and untreated with any topical drugs. Two eyes were under one to two kinds of antiglaucoma topical medication. The POAG and NTG were diagnosed by Glaucoma specialists. The diagnosis for POAG was based on the characteristic glaucomatous optic neuropathy, two reliable visual field tests with repeatable glaucomatous defect, open angle on gonioscopy and IOP more than $21 \mathrm{mmHg}$ on two consecutive visits using a Goldman applanation tonometer. The diagnosis for NTG was based on same criteria with IOP lower than $21 \mathrm{mmHg}$. Inclusion criteria were: more than 18 years old, diagnosis of POAG or NTG, willing to participate and being able to finish the CCM examination. Exclusion criteria were: secondary open angle glaucoma, angle closure, any corneal diseases like inflammation, leucoma and pterygium, any retinal diseases like retinal atrophy, bleeding or edema, .and Diabetes and other central neurologic disorders (Parkinson's disease, 
amyotrophic lateral sclerosis et al) with known corneal nerve alterations. Thirty-one eyes of 18 healthy control subjects who presented at the hospital for routine examination were enrolled as control group. The control group had no eye diseases other than low refractive error (myopia lower than 2D and astigmatism lower than 1D), and with ages more than 18 years old. Written consent was obtained from all participants, and the study was approved by the Ethical Committee of Peking University Third hospital. Investigations were conducted in accordance with the tenets of the Declaration of Helsinki.

All participants underwent a complete ophthalmic examination including a detailed slit lamp evaluation of the anterior segment, retinoscopy, CCM (Heidelberg Retina Tomograph『 Rostock Cornea Module, Heidelberg, Germany) and OCT (Zeiss Cirrus HD-OCT, Model 4000, Carl Zeiss Meditec, Inc.).

Following previously published procedures, CSNP around the central cornea was scanned with CCM at the depth of 35-50 $\mu \mathrm{m}$ using the sequence mode and the working area of $400 \times 400 \mu \mathrm{m}$. Each eye was anesthetized by 0.4\% Oxybuprocaine drops before examination and during the procedure the participants were asked to keep sitting position and fixate on a target light to assure scanning central corneas. A disposable sterile cap was set on the objective lens and ophthalmic gel was applied as a coupling medium between the lens cap and the cornea. At least 10 images of CSNP were obtained for each subject and 3-5 high quality images with no overlapping were selected for analysis. The scans were all performed by one experienced operator masked for patient's history and grouping 20 .

The CSNP was quantitatively analyzed using Neuron $\mathrm{J}$ software (an image $\mathrm{J}$ plugin, available online at image J.net). The assessment included: corneal nerve fiber length (FL), branch number (BN), corneal nerve fiber width (FW),corneal nerve fiber reflectivity(FR), nerve tortuosity (NT) and the existence of type 1, type 2 and total LCs. The nerve FL was defined as the total length of all nerve fibers and branches per image. Nerve BN was defined as the total number of branches emanating from major nerve trunks per image. Nerve FW was the width of the major nerve trunk calculated as a mean of 9 measurements for each image. Nerve FR was the average brightness of the nerve fiber per image which could be automatically acquired by the software. NT was measured by two methods. Local NT was defined as the local angle the nerve trunk formed and was calculated as the average of 9 measurements for each image. Total NT was defined as the ratio of the nerve trunk length to the straight distance between the two ends of it which indicated the bending of the nerve, and was also calculated as the average of 3 measurements for each image. The identification of type 1, type 2 and total LCs was recorded. According to previous descriptions, the type 1 LC was LCs with elongations and type 2 LC as round cells lacking dendrites ${ }^{21}$. Each parameter was a mean value of all 3-5 images selected for every eye.

Peripapillary RNFL thickness was measured for the POAG, NTG and normal by OCT. Optic nerve head was scanned to determine the RNFL thickness map. The temporal, superior, nasal and inferior quadrants of peripapillary RNFL were analyzed.

Statistical analysis was carried out using SPSS ( Statistical Package for Social Sciences) Windows version 20.0. Data were presented as mean \pm SD. The differences among the three groups were tested with analysis of variance (ANOVA) for age and with Chisquare for sex. Differences in opthalmological measures (FL, NB, NW, NR and NT) between two groups were assessed with independent-samples T Test. Chisquare was used for LCs analysis. The correlations of CSNP parameters with RNFL thickness in all three groups and with medication duration in the POAG group were evaluated using Pearson correlation coefficient. The $p$ value less than 0.05 was considered statistically significant. 


\section{Results}

\section{Patients characteristics}

The demographic data and clinical characteristics of all participants are presented in Table 1. There were no significant differences in mean age and gender distribution among the NTG, POAG and normal groups $(P=0.375$ for age and $P=0.212$ for gender). Nine eyes of POAG and eight eyes of NTG were newly diagnosed and had not started medicine treatment when enrolled, and they did not have significant difference in age and gender distribution either $(P=0.485$ for age and $P=0.707$ for gender). Neither did the POAG patients with and without treatment $(P=0.544$ for age and $P=0.907$ for gender). The medication time for the POAG patients (prostaglandin analogs only or combined with beta-blockers) ranged from 1 to 50 months with the average of $17.86 \pm 16.66$ months.

\section{Comparison of the CCM parameters of CSNP and LCs among the POAG, the NTG and the normal}

Firstly, we analyzed all patients including treated and untreated as well as the healthy subjects. The NTG group had the longest nerve FL of $3494.60 \pm 523.01 \mu \mathrm{m}$ while it was $3156.79 \pm 643.92 \mu \mathrm{m}$ in the POAG and $3223.78 \pm$ $617.06 \mu \mathrm{m}$ in the normal. However, no significant difference was identified in the value of nerve FL among the three groups (Table 2). NTG also had the most BN (19.78 \pm 5.56$)$, and both POAG (17.55 \pm 5.62$)$ and NTG groups had more nerve $B N$ than the normal $(13.38 \pm 5.44)$ with the $P$ values of 0.008 and 0.003 respectively. The NTG group had the thinnest FW of $2.96 \pm 1.20 \mu \mathrm{m}$ and the POAG group had the thickest of $3.65 \pm 0.69 \mu \mathrm{m}$. The difference between the two groups was significant $(p=0.043)$. The POAG had the highest FR of $153.09 \pm 14.12$, much brighter than the NTG $(143.03 \pm 14.01)$ and the normal $(143.91 \pm 15.91)(p=0.069$, and $p=0.033$ respectively). The NTG had the highest total NT $(1.09 \pm 0.07)$ and the lowest local NT (125.10 \pm 11.15$)$ indicating the most bending and tortuous trend of the corneal nerve in the NTG. The normal subjects had the lowest total NT $(1.06 \pm 0.03)$ and the highest local NT $(132.07 \pm 11.00)$ suggesting a relatively straight direction of the nerve. The total and local NT of POAG $(1.07 \pm 0.03$ and $130.56 \pm 13.03)$ were in the middle of the NTG and the control (Table 2).

It showed no difference among three groups as for total LC, neither for the type $1 \mathrm{LC}$ which was the mature form of the cell. However, type 2 LC existed in more POAG and NTG patients than in the control and the differences were significant $(p=0.024, p=0.027$ respectively).

Secondly, we analyzed each CNSP parameters between the untreated NTG and untreated POAG patients (both marked with $\mathrm{m}$-) to exclude the potential impact of antiglaucoma eye drops.

The NTG m- group had the longest nerve FL of all three groups with a length of $3646.10 \pm 457.09 \mu \mathrm{m}$, while it was $3026.25 \pm 684.28 \mu \mathrm{m}$ in the POAG $\mathrm{m}$ - and $3223.78 \pm 617.06 \mu \mathrm{m}$ in the normal. The differences were 0.047and 0.079 respectively. NTG $\mathrm{m}$ - group also had more BN $(20.75 \pm 5.86)$ than the other two groups, and the P values were 0.089 and 0.002 for POAG $\mathrm{m}$ - and the control respectively. The NTG m- had the thinnest FW of $2.52 \pm$ $0.81 \mu \mathrm{m}(\mathrm{p}=0.007$ and $p=0.018$ for the control and POAG $\mathrm{m}$ - respectively). The NTG $\mathrm{m}$ - had the lowest NR of $139.50 \pm 13.30$ while it was $143.91 \pm 15.91$ for the normal and $150.70 \pm 14.89$ for the POAG $\mathrm{m}$-, but the difference was not significant among the three. Though POAG m- group had more BN, NW and NR than the normal the difference was not significant. The NTG $m$ - had the highest total NT $(1.10 \pm 0.07)$ and the lowest local NT (121.56 \pm 9.35 ) also suggesting a tortuous feature. The POAG $\mathrm{m}$ - showed similar NT to the control (Table 3 ). 
The POAG $\mathrm{m}$ - and the NTG $\mathrm{m}$ - patients had more type $2 \mathrm{LC}$ than the control $(p=0.08, p=0.052$ respectively). The NTG $\mathrm{m}$ - also showed more type 1 LC and total LC and the difference was significant when compared with the POAG $m-(p=0.03$ and $p=0.024$ respectively $)$.

From the above results, the CSNP of NTG group no matter with or without medication could be described as thin and tortuous nerves with many branches and lower reflectivity. The POAG no matter with or without medication had CSNP more similar to that of the control with higher nerve reflectivity, wider nerve width, less nerve length, and less nerve branches and had a more straight trend than the NTG. Both the NTG and POAG patients had more type 2 LC than the normal (Fig. 1).

\section{Comparison of CCM parameters of CSNP and LCs in treated and untreated POAG}

We analyzed each CSNP parameters between the treated (marked as $\mathrm{m}+$ ) and untreated (marked as $\mathrm{m}-$ ) eyes in POAG group. Both POAG $\mathrm{m}$ + and $\mathrm{m}$ - patients had more nerve branches, wider nerve width, higher reflectivity than the control. However, the difference between POAG m- and the control group did not reach statistical significance (Table 2). The difference between POAG $m+$ and the control was significant for $B N$ and $N R(p=0.002, p=0.036$ respectively). Though POAG $m+$ had longer nerve FL, more BN, FW, FR, more nerve tortuosity than POAG $m$-, the difference was not significant (Table 2).

POAG $m+$ and POAG $m$ - had more type2 LC than the control ( $p=0.034$ and $p=0.08$ respectively), but the type 1 and total LC had no significant difference among three groups.

\section{Correlation between CCM parameters and medication duration in the POAG}

The correlation between each CCM parameters and the duration of anti-glaucoma medicine treatment in the POAG patients was analyzed and no significant association could be seen (Table 3).

\section{Correlation analysis between CCM parameters and OCT findings in the POAG, the NTG and the normal}

The correlation of peripapillary RNFL thickness and the corneal nerve parameters were analyzed in the POAG, NTG and the normal groups.

In POAG group, the nerve FL was associated with the superior quadrant of RNFL thickness $(r=0.547, p=0.043)$. Both the superior and nasal quadrants of RNFL thickness were statistically correlated with nerve BN. (nasal: $r=$ $0.568, P=0.034$; superior: $r=0.535, P=0.049)$. The brightness of nerve FR had significant correlations with all but the nasal quadrant of RNFL thickness (Table 4). The nerve FW and NT were not associated with the thickness of peripapillary RNFL. Neither was the LC existence.

All but the temporal quadrants of RNFL thickness were statistically correlated with nerve BN in the NTG (Table 4). The total NT had significant correlation with the inferior and the nasal quadrants (inferior: $r=-0.829, p=0.042$; nasal: $r=-0.972, r=0.001)$. The type 2 LC existence was associated with the superior quadrant of peripapillary RNFL thickness $(r=-0.878, p=0.021)$.

None but the inferior quadrants of RNFL thickness was statistically correlated with NR in the control (Table 4). No other correlations were identified.

\section{Discussion}


NTG has challenged the traditional pathophysiological viewpoints of glaucoma ever since it was recognized in $1900^{22}$. Occurring of retinal nerve fibers thinning and optic nerve atrophy in the absence of elevated IOP aroused a presumption that NTG could be classified as a disease of the neural system as well as the eye ${ }^{23}$. Evidence has shown a close pathogenetic link between NTG and degenerative diseases of central neural system such as Parkinson's disease and Alzheimer's disease ${ }^{24,25}$. The characteristic morphology of corneal nerves found in NTG patients in our research which was quite different from the features seen in the POAG and the control provided additional information to NTG's peripheral neural alterations.

CSNP is a good representative of peripheral small nerve fiber ${ }^{26}$. Thanks to the transparent and richly innervated nature of the cornea, images of CSNP noninvasively captured by in vivo CCM is now widely used in studies regarding neuropathy. The corneal nerve fiber length and nerve branches which are the two most commonly used CCM parameters have been reported to be associated with disease severity in some neural disorders such as diabetic neuropathy, Parkinson's disease and multiple sclerosis ${ }^{27-29}$. Evolving evidence suggests that the corneal nerve network might reflect the peripheral and central neurodegeneration. The corneal nerve in POAG and NTG is rarely studied though both central and peripheral nerve damage in POAG and NTG have been reported.

The results of our research present some morphological characteristics of CSNP in NTG and POAG and an obvious difference could be seen. The NTG group showed thin and tortuous corneal nerves with more branches and lower reflectivity, while the corneal nerve in the POAG was wider and brighter with less nerve length and less nerve branches. The POAG and the normal group had similar NT which was straighter than the NTG. To exclude the potential impact of antiglaucoma eye drops, we also analyzed the newly diagnosed POAG and NTG patients who had not received topical medication treatment, and the results were the same as above. This provided another supportive information that NTG might be a different disease entity from POAG.

Giorgio $A$ and his colleagues ${ }^{30}$ reported neurodegenerative findings across brain in NTG and similar but more severe changes in POAG. They suggested that glaucoma had degeneration in central nerve system that were partly independent of intraocular pressure and raised intraocular pressure in POAG represented a worsening factor for brain abnormalities. In terms of peripheral nerves, our findings indicated that the corneal nerve parameters of POAG lay in the middle between NTG and normal and were more similar to the normal especially in untreated POAG. Ranno $S$ and colleagues also found similar corneal confocal findings between normal subjects and untreated POAG glaucoma patients and concluded that their untreated glaucoma patients and controls had similar corneal parameters ${ }^{31}$. However, Ranno $S$ found significant difference between treated and untreated POAG in corneal nerves morphology which is contrary to our findings. We found no difference in CCM parameters between treated and untreated POAG. The explanation might be that in Ranno's research patients should use the antiglaucoma eye drops for at least 2 years but in our research the medication duration is much less. Longstanding medical glaucoma treatment may cause corneal nerve alteration ${ }^{32}$. More participants should be included for further confirmation.

Would the specific nerve pattern of NTG shown in our research indicate a primary corneal nerve alteration which was independent of intraocular pressure? Corneal biomechanics and central corneal thickness were analyzed in NTG but few investigations have been made on corneal nerve in NTG. ${ }^{33}, 34$. A whorl-like corneal subbasal nerve plexus in normal eyes was depicted by Utsunomiya T's report which was in the inferocentral area of the cornea 27. However, the tortuosity observed in our NTG patients was not whorl like (Fig. 1) but a zigzag pattern and the area we examined was in the center instead of the inferocenter. So the winding nerves seen in our result was not 
because of incorrect location nor a shift of nerve distribution. It was more likely a primary alteration and thus supported the hypothesis that NTG is a primary degenerative neuropathy independent of IOP.

Both the NTG and POAG in our research had more type 2 LC than the normal. The type 2 LCs existed rarely in the normal (3/31), but more common in POAG (untreated 3/9, treated 7/14)and NTG (untreated 3/8) patients though the difference between the normal and untreated POAG/NTG did not reach significance $(p=0.08$, and $p=0.052$ for POAG $\mathrm{m}$ - and NTG $\mathrm{m}$ - respectively, $\mathrm{p}=0.034$ for POAG $\mathrm{m}+$ ). LCs are antigen-presenting cells residing exclusively in the corneal epithelium in the vicinity of corneal subbasal nerves and can be identified as bright, mostly oval or elongated particles with a diameter of up to $15 \mu \mathrm{m}^{35}$. LCs are crucial for immune responses and modulations. According to a previous description, Type 2 LC which lacks the processes is the immature form ${ }^{36}$. The elevation of type 2 LCs in POAG and NTG might indicate a persistent migration and maturation of this immune cells. Marsovszky $L^{36}$ analyzed the antihypertention topical medications containing two different preservatives and found LC densities were greater in both glaucoma groups compared to control subjects. He then concluded a detrimental effect of the preservatives to the cornea. However, the author did not analyze untreated POAG and no previous confocal assessment was made on corneal LCs of untreated glaucoma patients. The possibility still exists that the gathering of LCs in both POAG and NTG is due to the disease itself (e.g. an immunologic process) and the drug exacerbated it. Significant difference between normal and the treated POAG and significant increase of type 2 LC were spotted when both treated and nontreated patients were included in the comparison indicating the topical medication exerted a worsening impact. Type 1 LCs and total LCs showed difference among the groups without statistical significance which might be due to small number of subjects. More participants would be investigated in further study.

What is the interference of the topical antihypertension medication to the corneal nerve indeed? Ranno $S$ compared nontreated POAG patients and those on medical treatment for at least two years and came up with a result that both corneal nerve tortuosity and reflectivity in treated patients were lower than in nontreated patients ${ }^{31}$. Martone $\mathrm{G}$ found a decrease of CSNP number and an increase of nerve tortuosity in medication treated POAG compared with the normal ${ }^{37}$. Our research showed a longer FL, more BN, wider NW, higher NR, more tortuosity and more LCs in treated POAG than untreated patients but the difference was not significant. And no significant correlation was found between medication time and the CCM parameters. Here raised another question, would these nerve alterations shown in the treated patients be attributed to the disease progress, the severity of the neurodegeneration instead of the drug's neural toxicity?

We then analyzed the correlations between RFNL and the CCM parameters and found that they had some associations. The CCM parameters in the normal had no correlations with RNFL thickness, but parameters such as nerve branches, reflectivity and nerve length in the POAG and branches number, total tortuosity and existence of type 2 LCs in the NTG were associated with RNFL thickness. The temporal quadrant had the least correlation with the CCM parameters in glaucoma patients.

The limitations of the current study were the small number of subjects and the lack of tear film status records as well as the functional evaluations of the peripheral nerves. As for the effect of the anti-glaucoma topical medication on corneal nerves in glaucoma patients, a well-designed study is in need to differentiate the process of neurodegeneration and the drug's impact. 
Our study showed that the corneal nerves in NTG patients have characteristic pattern different from that in the POAG and the normal, while the latter two share more morphological features. And the nerve parameters in CCM especially BN have associations with the RNFL thickness, a marker for disease progression in those glaucoma patients. The results added supportive information to the pathological link between NTG and the neural system alteration. Also it provoked the re-thinking of NTG's entity adscription. Besides the nerve alterations, both untreated POAG and NTG have obvious immature LCs infiltration indicating that the glaucoma disease itself not the antiglaucoma mediation had some relationship to ocular surface inflammation. The antiglaucoma topical medications might have worsening impact on corneal nerves and LCs infiltration, but it should be further distinguished from a probable primary corneal neurodegeneration in the glaucoma patients.

\section{Conclusion}

The NTG patients had unique nerve morphology in CSNP which was quite different from that in the POAG and the normal, while the latter two had more common features. The CCM parameters might be potential indicators for glaucoma prognosis as they were associated with the RNFL thickness. Untreated glaucoma patients had obvious immature LCs in cornea indicating their relationship with ocular surface inflammation.

\section{Abbreviations}

POAG

Primary open angle glaucoma

RNFL

retinal nerve fiber layer

IOP

intraocular pressure

RGC

retinal ganglion cells

NTG

normal tension glaucoma

CCM

corneal confocal microscopy

CSNP

corneal subbasal nerve fiber plexus

FL

fiber length

BN

branch number

FW

fiber width

FR

fiber reflectivity

NT

nerve tortuosity 


\section{Declarations}

- Ethics approval and consent to participate: the study was approved by the Ethical Committee of Peking University Third hospital.

- Consent for publication: all authors and our institution have approved the publication

- Availability of data and materials: Not applicable

- Competing interests: none

- Funding: none

- Authors' contributions: ZY Liu and DL Jing: interpretation of data and drafting the work, YL Chou, HK Wang and S Gao: acquisition of data, W Wang: design of the work and revising the work. All authors have read and approved the manuscript.

- Acknowledgements: Dr. H Zhang is a statistician working in Peking University third hospital and helped us with statistical consultation for this manuscript.

\section{References}

1. Faralli JA, Filla MS, Peters DM. Role of Fibronectin in Primary Open Angle Glaucoma. Cells-Basel. 2019;8(12).

2. Tham YC, Li X, Wong TY, Quigley HA, Aung T, Cheng CY. Global prevalence of glaucoma and projections of glaucoma burden through 2040: a systematic review and meta-analysis. Ophthalmology. 2014;121(11):2081-2090.

3. Risner ML, McGrady NR, Pasini S, Lambert WS, Calkins DJ. Elevated ocular pressure reduces voltage-gated sodium channel NaV1.2 protein expression in retinal ganglion cell axons. Exp Eye Res. 2020;190:107873.

4. Calkins DJ. Critical pathogenic events underlying progression of neurodegeneration in glaucoma. Prog Retin Eye Res. 2012;31(6):702-719.

5. Kim KE, Park KH. Update on the Prevalence, Etiology, Diagnosis, and Monitoring of Normal-Tension Glaucoma. Asia Pac J Ophthalmol (Phila). 2016;5(1):23-31.

6. Trivli A, Koliarakis I, Terzidou C, et al. Normal-tension glaucoma: Pathogenesis and genetics. Exp Ther Med. 2019;17(1):563-574.

7. Mastropasqua R, Fasanella V, Agnifili L, et al. Advance in the pathogenesis and treatment of normal-tension glaucoma. Prog Brain Res. 2015;221:213-232.

8. Chiotoroiu SM, Stefaniu O, Noaghi M, Teodorescu A, Taina L. THE ROLE OF SYSTEMIC BLOOD PRESSURE IN GLAUCOMA PROGRESSION. Rom J Ophthalmol. 2015;59(3):141-147.

9. Choi J, Kook MS. Systemic and Ocular Hemodynamic Risk Factors in Glaucoma. Biomed Res Int. 2015;2015:141905.

10. Mozaffarieh M, Flammer J. New insights in the pathogenesis and treatment of normal tension glaucoma. Curr Opin Pharmacol. 2013;13(1):43-49.

11. Rizzo MI, Greco A, De Virgilio A, et al. Glaucoma: recent advances in the involvement of autoimmunity. Immunol Res. 2017;65(1):207-217.

12. Giorgio A, Zhang J, Costantino F, De Stefano N, Frezzotti P. Diffuse brain damage in normal tension glaucoma. Hum Brain Mapp. 2018;39(1):532-541. 
13. Kremmer S, Kreuzfelder E, Bachor E, Jahnke K, Selbach JM, Seidahmadi S. Coincidence of normal tension glaucoma, progressive sensorineural hearing loss, and elevated antiphosphatidylserine antibodies. $\mathrm{Br} J$ Ophthalmol. 2004;88(10):1259-1262.

14. Omoti AE, Edema OT. A review of the risk factors in primary open angle glaucoma. Niger $J$ Clin Pract. 2007;10(1):79-82.

15. Aksoy D, Ortak H, Kurt S, Cevik E, Cevik B. Central corneal thickness and its relationship to Parkinson's disease severity. Can J Ophthalmol. 2014;49(2):152-156.

16. Hubers A, Muller HP, Dreyhaupt J, et al. Retinal involvement in amyotrophic lateral sclerosis: a study with optical coherence tomography and diffusion tensor imaging. J Neural Transm (Vienna). 2016;123(3):281287.

17. Zis P, Grunewald RA, Chaudhuri RK, Hadjivassiliou M. Peripheral neuropathy in idiopathic Parkinson's disease: A systematic review. J Neurol Sci. 2017;378:204-209.

18. Dehghani C, Pritchard N, Edwards K, Russell AW, Malik RA, Efron N. Risk Factors Associated With Corneal Nerve Alteration in Type 1 Diabetes in the Absence of Neuropathy: A Longitudinal In Vivo Corneal Confocal Microscopy Study. Cornea. 2016;35(6):847-852.

19. Liu Z, Wang H, Fan D, Wang W. Comparison of optical coherence tomography findings and visual field changes in patients with primary open-angle glaucoma and amyotrophic lateral sclerosis. $J$ Clin Neurosci. 2018;48:233-237.

20. Villani E, Sacchi M, Magnani F, et al. The Ocular Surface in Medically Controlled Glaucoma: An In Vivo Confocal Study. Invest Ophthalmol Vis Sci. 2016;57(3):1003-1010.

21. Postole AS, Knoll AB, Auffarth GU, Mackensen F. In vivo confocal microscopy of inflammatory cells in the corneal subbasal nerve plexus in patients with different subtypes of anterior uveitis. Br J Ophthalmol. 2016;100(11):1551-1556.

22. Killer HE, Pircher A. Normal tension glaucoma: review of current understanding and mechanisms of the pathogenesis. Eye (Lond). 2018;32(5):924-930.

23. Zhang HJ, Mi XS, So KF. Normal tension glaucoma: from the brain to the eye or the inverse? Neural Regen Res. 2019;14(11):1845-1850.

24. Eraslan M, Cerman E, Cekic O, et al. Neurodegeneration in ocular and central nervous systems: optical coherence tomography study in normal-tension glaucoma and Alzheimer disease. Turk J Med Sci. 2015;45(5):1106-1114.

25. Garcia-Martin E, Satue M, Fuertes I, et al. Ability and reproducibility of Fourier-domain optical coherence tomography to detect retinal nerve fiber layer atrophy in Parkinson's disease. Ophthalmology. 2012;119(10):2161-2167.

26. Khan A, Petropoulos IN, Ponirakis G, et al. Corneal confocal microscopy detects severe small fiber neuropathy in diabetic patients with Charcot neuroarthropathy. J Diabetes Investig. 2018;9(5):1167-1172.

27. Utsunomiya T, Nagaoka T, Hanada K, et al. Imaging of the Corneal Subbasal Whorl-like Nerve Plexus: More Accurate Depiction of the Extent of Corneal Nerve Damage in Patients With Diabetes. Invest Ophthalmol Vis Sci. 2015;56(9):5417-5423.

28. Mikolajczak J, Zimmermann H, Kheirkhah A, et al. Patients with multiple sclerosis demonstrate reduced subbasal corneal nerve fibre density. Mult Scler. 2017;23(14):1847-1853. 
29. Kass-lliyya L, Javed S, Gosal D, et al. Small fiber neuropathy in Parkinson's disease: A clinical, pathological and corneal confocal microscopy study. Parkinsonism Relat Disord. 2015;21(12):1454-1460.

30. Giorgio A, Zhang J, Costantino F, De Stefano N, Frezzotti P. Diffuse brain damage in normal tension glaucoma. Hum Brain Mapp. 2018;39(1):532-541.

31. Ranno S, Fogagnolo P, Rossetti L, Orzalesi N, Nucci P. Changes in corneal parameters at confocal microscopy in treated glaucoma patients. Clin Ophthalmol. 2011;5:1037-1042.

32. Zhang X, Vadoothker S, Munir WM, Saeedi O. Ocular Surface Disease and Glaucoma Medications: A Clinical Approach. Eye Contact Lens. 2019;45(1):11-18.

33. Park K, Shin J, Lee J. Relationship between corneal biomechanical properties and structural biomarkers in patients with normal-tension glaucoma: a retrospective study. Bmc Ophthalmol. 2018;18(1):7.

34. Lee JW, Wong RL, Chan JC, Wong IY, Lai JS. Differences in corneal parameters between normal tension glaucoma and primary open-angle glaucoma. Int Ophthalmol. 2015;35(1):67-72.

35. Hattori T, Chauhan SK, Lee H, et al. Characterization of Langerin-expressing dendritic cell subsets in the normal cornea. Invest Ophthalmol Vis Sci. 2011;52(7):4598-4604.

36. Marsovszky L, Resch MD, Visontai Z, Nemeth J. Confocal microscopy of epithelial and langerhans cells of the cornea in patients using travoprost drops containing two different preservatives. Pathol Oncol Res. 2014;20(3):741-746.

37. Martone G, Frezzotti P, Tosi GM, et al. An in vivo confocal microscopy analysis of effects of topical antiglaucoma therapy with preservative on corneal innervation and morphology. Am J Ophthalmol. 2009;147(4):725-735.

\section{Tables}

Table 1. The Demographic Data and Clinical Characteristics of All Subjects

\begin{tabular}{|c|c|c|c|c|c|c|c|}
\hline & \multicolumn{3}{|c|}{ POAG } & \multicolumn{3}{|c|}{ NTG } & \multirow[t]{2}{*}{ Control } \\
\hline & Total & $\mathrm{m}+$ & m- & Total & $\mathrm{m}+$ & m- & \\
\hline $\mathrm{n}$ & 23 & 14 & 9 & 10 & 2 & 8 & 31 \\
\hline Age (year) & $56.74 \pm 15.45$ & $58.36 \pm 12.43$ & $54.22 \pm 19.85$ & $60.80 \pm 5.98$ & - & $59.25 \pm 5.68$ & $53.10 \pm 17.84$ \\
\hline $\mathrm{p}$-value & & $0.544 \mathrm{~b}$ & & & & $0.485 \mathrm{c}$ & $0 . \overline{375 a}$ \\
\hline Female sex-no (\%) & $15(65 \%)$ & $9(64 \%)$ & $6(67 \%)$ & $6(60 \% \square$ & & $6(75 \%)$ & $13(42 \%$ 口 \\
\hline $\mathrm{p}$ value & & $0.907 \mathrm{~b}$ & & & & $0.707 \mathrm{c}$ & $0.212 \mathrm{a}$ \\
\hline m+-no(\%) & $14(61 \%)$ & & & $8(80 \%)$ & & & \\
\hline $\mathrm{p}$ value & $0.031 \mathrm{~d}$ & & & & & & \\
\hline Medication duration (month) & & $17.86 \pm 16.66$ & & & & & \\
\hline
\end{tabular}

a: $p$ value of comparison among POAG, NTG and control; b: $p$ value of comparison between POAG m+ and m-; c: $p$ value of comparison between POAG m- and NTG m-; d: p value of comparison between POAG and NTG.

$\mathrm{m}$-: patients without topical medication; $\mathrm{m}+$ : patients with topical medication 
Table 2. Comparison of Confocal Parameters Between the normal tension glaucoma, primary open angle glaucoma and the normal

\begin{tabular}{|c|c|c|c|c|c|c|c|c|c|c|c|}
\hline & & $\mathrm{n}$ & FL & $\mathrm{BN}$ & NW & NR & $\begin{array}{l}\text { NT } \\
\text { total }\end{array}$ & $\begin{array}{l}\text { NT } \\
\text { local }\end{array}$ & $\begin{array}{l}\text { LC Type } \\
1 \\
\text { n( } \\
\%)\end{array}$ & $\begin{array}{l}\text { LC } \\
\text { Type2 } \\
\%)\end{array}$ & $\begin{array}{l}\text { LC total } \\
\text { n ( \%) }\end{array}$ \\
\hline Normal & & 31 & $3223.78 \pm 617.06$ & $13.38 \pm 5.44$ & $3.37 \pm 0.74$ & $143.91 \pm 15.91$ & $1.06 \pm 0.03$ & $132.07 \pm 11.00$ & $14(45.16)$ & $3 /(9.68)$ & $16(51.61)$ \\
\hline \multirow[t]{6}{*}{ POAG } & total & 23 & $3156.79 \pm 643.92$ & $17.55 \pm 5.62$ & $3.65 \pm 0.69$ & $153.09 \pm 14.12$ & $1.07 \pm 0.03$ & $130.56 \pm 13.03$ & $9(39.13)$ & $8(34.78)$ & $11(47.83)$ \\
\hline & $\begin{array}{l}P \text { value } \\
\text { Vs control }\end{array}$ & & 0.700 & $0.008^{*}$ & 0.160 & $0.033^{*}$ & 0.683 & 0.647 & 0.658 & $0.024^{*}$ & 0.783 \\
\hline & $\begin{array}{l}P \text { value } \\
\text { Vs control }\end{array}$ & & 0.933 & $0.002 *$ & 0.193 & $0.036^{*}$ & 0.590 & 0.252 & 0.763 & $0.034^{*}$ & 0.731 \\
\hline & $\begin{array}{l}P \text { value } \\
\text { Vs } \\
\text { POAGm- }\end{array}$ & & 0.448 & 0.116 & 0.928 & 0.527 & 0.719 & 0.246 & 0.183 & 0.907 & 0.265 \\
\hline & m- & 9 & $3026.25 \pm 684.28$ & $15.24 \pm 6.55$ & $3.64 \pm 0.91$ & $150.70 \pm 14.89$ & $1.06 \pm 0.03$ & $134.57 \pm 15.78$ & $2(22.22)$ & $3(33.33)$ & $3(33.33)$ \\
\hline & $\begin{array}{l}P \text { value } \\
\text { Vs control }\end{array}$ & & 0.414 & 0.394 & 0.374 & 0.261 & 0.971 & 0.665 & 0.216 & 0.08 & 0.334 \\
\hline \multirow[t]{6}{*}{ NTG } & total & 10 & $3494.60 \pm 523.01$ & $19.78 \pm 5.56$ & $2.96 \pm 1.20$ & $143.03 \pm 14.01$ & $1.09 \pm 0.07$ & $125.10 \pm 11.15$ & $6(60.00)$ & $4(40.00)$ & $8(80.00)$ \\
\hline & $\begin{array}{l}P \text { value } \\
\text { Vs control }\end{array}$ & & 0.219 & $0.003 *$ & 0.201 & 0.876 & $0.036^{*}$ & 0.090 & 0.414 & $0.027 *$ & 0.113 \\
\hline & $\begin{array}{l}P \text { value } \\
\text { Vs POAG }\end{array}$ & & 0.155 & 0.302 & $0.043^{*}$ & 0.069 & 0.074 & 0.258 & 0.269 & 0.775 & 0.086 \\
\hline & m- & 8 & $3646.10 \pm 457.09$ & $20.75 \pm 5.86$ & $2.52 \pm 0.81$ & $139.50 \pm 13.30$ & $1.10 \pm 0.07$ & $121.56 \pm 9.35$ & $6(75.00)$ & $3(37.50)$ & $7(78.50)$ \\
\hline & $\begin{array}{l}P \text { value } \\
\text { Vs control }\end{array}$ & & 0.079 & $0.002^{*}$ & $0.007^{*}$ & 0.476 & $0.020^{*}$ & $0.018^{*}$ & 0.132 & 0.052 & 0.066 \\
\hline & $\begin{array}{l}P \text { value } \\
\text { Vs } \\
\text { POAGm- }\end{array}$ & & $0.047^{*}$ & 0.089 & $0.018^{*}$ & 0.125 & 0.153 & 0.06 & $0.03^{*}$ & 0.858 & $0.024^{*}$ \\
\hline
\end{tabular}

* denotes a statistically significant difference

POAG: primary open angle glaucoma

NTG: normal tension glaucoma

m-: patients without topical medication; $\mathrm{m}+$ : patients with topical medication

Table 3. Correlations between medication duration and the Confocal parameters in the treated primary open angle glaucoma

\begin{tabular}{llllllllll}
\hline & FL & BN & NW & NR & Total NT & Local NT & LC Type 1 & LC Type2 & LC total \\
\hline r & -0.169 & -0.415 & -0.388 & 0.146 & 0.042 & -0.455 & 0.143 & -0.261 & 0.054 \\
P value & 0.565 & 0.140 & 0.170 & 0.618 & 0.886 & 0.102 & 0.626 & 0.367 & 0.854 \\
\hline
\end{tabular}

\begin{tabular}{|c|c|c|c|c|c|c|c|c|c|}
\hline & FL & $\mathrm{BN}$ & NW & NR & $\begin{array}{l}\text { Total NT } \\
\text { r/P value }\end{array}$ & Local NT & LC Type 1 & LC Type 2 & LC \\
\hline \multicolumn{10}{|l|}{ POAG } \\
\hline Superior & $0.547 / 0.043^{*}$ & 0.535/0.049* & $0.270 / 0.350$ & $-0.741 / 0.002 *$ & $-0.112 / 0.703$ & $-0.125 / 0.670$ & $-0.129 / 0.659$ & $-0.021 / 0.944$ & $-0.021 / 0.944$ \\
\hline Inferior & $0.279 / 0.334$ & $0.322 / 0.262$ & $0.015 / 0.958$ & $-0.749 / 0.002 *$ & $0.182 / 0.577$ & $0.037 / 0.899$ & $0.240 / 0.408$ & $0.358 / 0.209$ & $0.358 / 0.209$ \\
\hline Nasal & $0.333 / 0.245$ & $0.568 / 0.034 *$ & $-0.320 / 0.265$ & $-0.086 / 0.770$ & $0.163 / 0.577$ & $-0.357 / 0.210$ & $0.371 / 0.192$ & $0.234 / 0.422$ & $0.234 / 0.422$ \\
\hline Temporal & $0.288 / 0.318$ & $0.207 / 0.478$ & $0.218 / 0.455$ & $-0.538 / 0.047^{*}$ & $0.037 / 0.899$ & $-0.305 / 0.288$ & $0.166 / 0.570$ & $0.286 / 0.321$ & $0.286 / 0.321$ \\
\hline \multicolumn{10}{|l|}{ NTG } \\
\hline Superior & $0.371 / 0.468$ & $-0.860 / 0.028 *$ & $-0.061 / 0.909$ & $0.446 / 0.375$ & $-0.600 / 0.208$ & $0.429 / 0.396$ & $0.007 / 0.990$ & $-0.878 / 0.021$ & $-0.131 / 0.805$ \\
\hline Inferior & $0.200 / 0.704$ & $-0.846 / 0.034 *$ & $-0.113 / 0.831$ & $0.402 / 0.430$ & $-0.829 / 0.042$ & $0.486 / 0.329$ & $-0.117 / 0825$ & $-0.683 / 0.135$ & $0.131 / 0.805$ \\
\hline Nasal & $-0.371 / 0.468$ & $-0.796 / 0.058$ & $0.572 / 0.235$ & $0.637 / 0.174$ & $-0.972 / 0.001$ & $0.600 / 0.208$ & $0.414 / 0.414$ & $-0.293 / 0.573$ & $0.655 / 0.158$ \\
\hline Temporal & $0.435 / 0.389$ & $0.207 / 0.693$ & $-0.151 / 0.776$ & $-0.620 / 0.190$ & $0.120 / 0.821$ & $-0.493 / 0.321$ & $-0.735 / 0.096$ & $0.099 / 0.852$ & $-0.266 / 0.611$ \\
\hline \multicolumn{10}{|l|}{ Normal } \\
\hline Superior & $-0.385 / 0.306$ & $-0.042 / 0.915$ & $-0.147 / 0.706$ & $-0.310 / 0.417$ & $0.268 / 0.486$ & $-0.477 / 0.194$ & $-0.043 / 0.912$ & $0.550 / 0.125$ & $-0.0 .043 / 0.912$ \\
\hline Inferior & $0.042 / 0.915$ & $0.286 / 0.456$ & $-0.042 / 0.914$ & $-0.840 / 0.005^{*}$ & $0.403 / 0.282$ & $0.378 / 0.316$ & $0.437 / 0.240$ & $0.552 / 0.123$ & $0.437 / 0.240$ \\
\hline Nasal & $0.544 / 0.130$ & $-0.059 / 0.881$ & $0.319 / 0.402$ & $-0.293 / 0.444$ & $-0.149 / 0.702$ & $-0.142 / 0.715$ & $-0.348 / 0.359$ & $-0.413 / 0.270$ & $-0.348 / 0.359$ \\
\hline Temporal & $-0.360 / 0.342$ & $0.017 / 0.966$ & $0.261 / 0.498$ & $0.109 / 0.781$ & $0.285 / 0.458$ & $0.452 / 0.222$ & $0.174 / 0.654$ & $0.481 / 0.190$ & $0.174 / 0.654$ \\
\hline
\end{tabular}

* denotes a statistically significant difference

FL: fiber length, BN: branch number, NW: nerve width, NR:nerve reflectivity, NT: nerve tortuosity, LCs: Langerhans cells

POAG: primary open angle glaucoma, including treated and untreated patients

NTG: normal tension glaucoma, including treated and untreated patients 


\section{Figures}

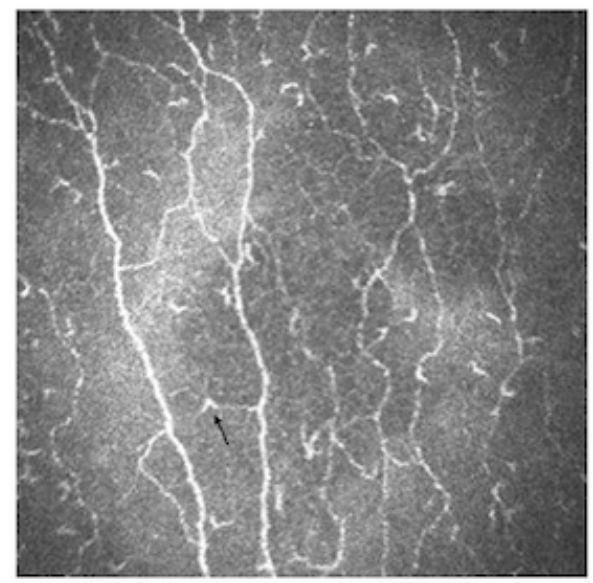

A

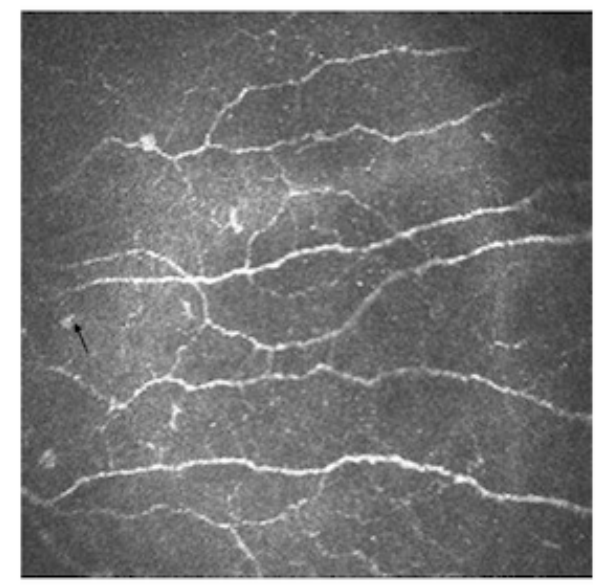

B

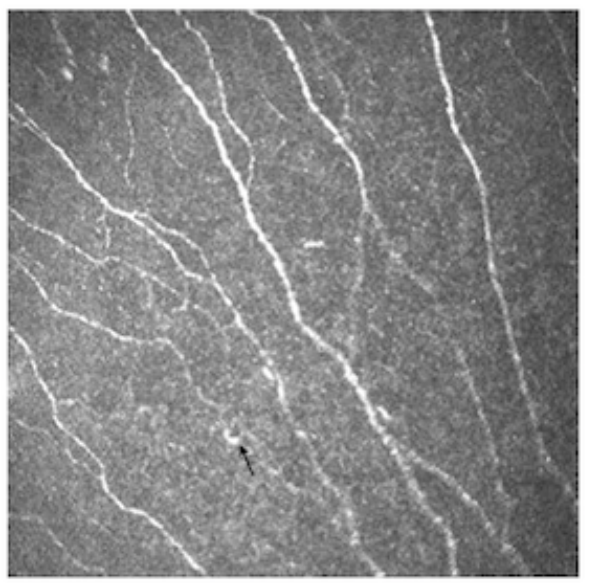

C

\section{Figure 1}

characteristics of the CSNP in the NTG, POAG and the control. A. Tortuous corneal nerves with more branches in a newly diagnosed NTG patient. B. less winding corneal nerves with less branches in a newly diagnosed POAG patient. C. the relatively straight nerves in a normal subject. Arrows notes the LCs. 